\title{
Bevacizumab-Induced Tumor Vasculature Normalization and Sequential Chemotherapy in Colorectal Cancer: An Interesting and Still Open Question
}

\author{
Alessandro Ottaiano ${ }^{1 *}$ and Michele Caraglia ${ }^{2}$ \\ ${ }^{1}$ Istituto Nazionale Tumori di Napoli, IRCCS "G. Pascale", Naples, Italy, ${ }^{2}$ Department of Precision Medicine, University \\ "L. Vanvitelli" of Naples, Naples, Italy
}

Keywords: colorectal cancer, bevacizumab, oxaliplatin, normalization window, VEGF

\section{OPEN ACCESS}

Edited by:

Giovanni Li Volti,

University of Catania, Italy

Reviewed by:

Roberto Bei,

University of Rome Tor Vergata, Italy

Maria Caterina Turco,

University of Salerno, Italy

*Correspondence:

Alessandro Ottaiano

a.ottaiano@istitutotumori.na.it

Specialty section:

This article was submitted to

Gastrointestinal Cancers:

Colorectal Cancer,

a section of the journal

Frontiers in Oncology

Received: 02 August 2021 Accepted: 06 September 2021 Published: 24 September 2021

Citation:

Ottaiano A and Caraglia M (2021) Bevacizumab-Induced Tumor Vasculature Normalization and

Sequential Chemotherapy in

Colorectal Cancer: An Interesting and Still Open Question.

Front. Oncol. 11:751986.

doi: 10.3389/fonc.2021.751986

\section{INTRODUCTION}

Bevacizumab is a recombinant fully humanized IgG1 targeting the Vascular Endothelial Growth Factor A (VEGF-A). The rationale of its use in oncology relies on the critical role of VEGF-Ainduced neo-angiogenesis in the growth of many solid tumors (1-3). Since its discovery, many evidences suggested that it did not display direct antitumor action but rather contributes to improve the effects of associated chemotherapy. In fact, following bevacizumab administration, intratumoral vessels become morphologically more organized, and hypoxia and interstitial fluid pressure reduce ("vasculature normalization"); as a consequence, chemotherapy drug delivery into tumor masses ameliorates (4). Avallone et al. published the first randomized phase III study (OBELICS study) comparing the sequential administration of bevacizumab before standard oxaliplatin-based chemotherapy versus a traditional concomitant regimen in metastatic colorectal cancer (CRC) (5). The intent was to optimize bevacizumab and chemotherapy association by administering the chemotherapy in the "normalization window" and ameliorate the antitumor effects. The "normalization window" is the time window during which the anarchist texture of tumor vasculature becomes macroscopically more linear and less dense (1-4) (Figure 1). The authors fail to meet the primary end-point based on a difference in objective response rate (ORR) between the two arms. The odds ratio of response for experimental (ORR: 56.5\%) versus standard arm (ORR: $57.4 \%$ ) was 0.96 ( $95 \%$ CI: $0.55-1,68, \mathrm{P}=0.89$ ). We would add insights and prompt discussion making hypotheses on the causes of failure. This is important for scientific discussion and for planning future trials.

\section{THE HETEROGENEITY OF "NORMALIZATION WINDOW" IN TIME AND SPACE}

The "normalization window" has an intrinsic heterogeneous nature in time and space. In fact, it is transient and closes as the anti-angiogenic drug effect cyclically reduces (Figure 1). A crucial 


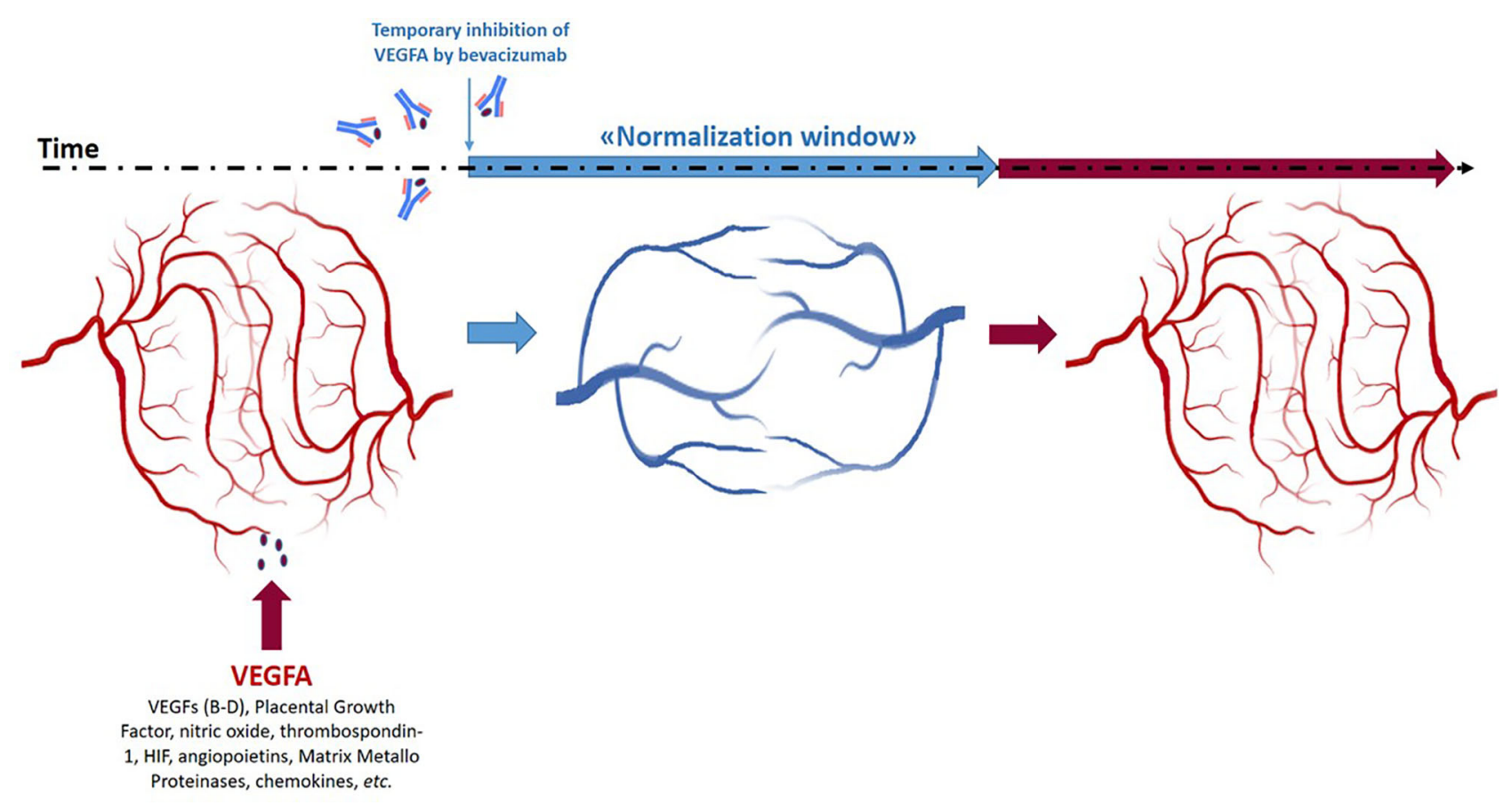

FIGURE 1 | Schematic representation of the "normalization window" of anti-angiogenic treatment of tumor masses during time and space.

question rises: which is the nadir of this effect? The theories prediction of this nadir should be integrated into study design to choose the best timing to administer chemotherapy. Unfortunately, this is still an unanswered question. Preclinical xenograft models with tumor cell lines are too homogeneous and far from the wild tumor human mechanisms. However, in some cases, the transient window is identified also earlier and later than 4 days timing used in the study by Avallone et al. (6). Most importantly, in clinical practice, with human subjects, the nadir of normalization is difficult to evaluate and is also physiologically heterogeneous (7-10). In a mathematical and intuitive modeling of the effect of anti-angiogenic drugs, Hutchinson et al. demonstrated that one of the most important parameters to identify the "normalization window" is the tumor volume rather than the number of metastatic sites accounted for a stratification factor in the randomization procedure of the OBELICS study. Hutchinson describes "normalization windows" ranging until 21 days (11).

\section{DISCUSSION}

Some considerations are raised that have not been considered by OBELICS investigators to interpret their results. Are 4 days sufficient to administer chemotherapy in a correct normalization window? The use of diffusion-weighted magnetic resonance (DWMR) imaging, which is widely accepted for monitoring intratumoral vasculature evolution, could have contributed to identify and "personalize" the normalization window. In fact, bevacizumab-induced tumor vasculature normalization should be evaluated and integrated into the design of future trials to adequately assess efficacy of sequential combination treatments (12). The authors' choice to administer bevacizumab 4 days before chemotherapy was predominantly based on a previous study (BRANCH trial) (13) in rectal cancer; however, this is questionable since both clinical and biological behaviors of colon and rectal cancers, including their molecular signatures, are profoundly different (14). It has also to be considered that the usage of bevacizumab can induce modifications in the assets of cytogenetics and factors involved in the regulation of angiogenesis. In fact, although it was reported that bevacizumab-induced increase in VEGF did not affect the sensitivity of tumors to anticancer treatments, it cannot be excluded that other angiogenic factors can be modulated during single-agent bevacizumab administration inferring the treatment activity (15). Thus, a biomarker-based stratification of the patients (i.e., high $v s$ low basal VEGF-A) should be required in order to evaluate the efficacy of the sequential administration. Moreover, it is reported that bevacizumab in CRC can determine profound metabolic changes peculiar of hypoxic conditions together with HIF (Hypoxia Inducible Factor) increased expression (16). This can, in turn, induce resistance to chemotherapy deserving HIF-blocking strategies to re-sensitize cancer to drugs (17).

In conclusion, we believe that the normalization effect of bevacizumab on tumor vasculature in CRC patients is a fascinating scientific question that remains to be determined as the optimization of the therapy through sequential administration with chemotherapy. In the future, we propose 
to integrate DWMR imaging in study design to adequately assess the "normalization window" and to perform biomarkers- and tumor volume-based stratification of patients for better interpretation of the results.

\section{AUTHOR CONTRIBUTIONS}

All authors listed have made a substantial, direct, and intellectual contribution to the work and approved it for publication.

\section{REFERENCES}

1. Folkman J. Angiogenesis in Cancer, Vascular, Rheumatoid Andother Disease. Nat Med (1995) 1:27-31. doi: 10.1038/nm0195-27

2. Ferrara N, Davis-Smyth T. The Biology of Vascular Endothelialgrowth Factor. Endocr Rev (1997) 18:4-25. doi: 10.1210/edrv.18.1.0287

3. Presta LG, Chen H, O'Connor SJ, Chisholm V, Meng YG, Krummen L, et al. Humanization of Anantivascular Endothelial Growth Factor Monoclonal Antibody for the Therapy Ofsolid Tumors and Other Disorders. Cancer Res (1997) 57:4593-9.

4. Ellis LM. Mechanisms of Action of Bevacizumab as a Component of Therapy for Metastatic Colorectal Cancer. Semin Oncol (2006) 33:S1-7. doi: 10.1053/ j.seminoncol.2006.08.002

5. Avallone A, Piccirillo MC, Nasti G, Rosati G, Carlomagno C, Di Gennaro E, et al. Effect of Bevacizumab in Combination With Standard OxaliplatinBased Regimens in Patients With Metastatic Colorectal Cancer: A Randomized Clinical Trial. JAMA Netw Open (2021) 4(7):e2118475. doi: 10.1001/jamanetworkopen.2021.184752

6. Dickson PV, Hamner JB, Sims TL, Fraga CH, Ng CY, Rajasekeran S, et al. Bevacizumab-Induced Transient Remodeling of the Vasculature in Neuroblastoma Xenografts Results in Improved Delivery and Efficacy of Systemically Administered Chemotherapy. Clin Cancer Res (2007) 13 (13):3942-50. doi: 10.1158/1078-0432.CCR-07-0278

7. Anzidei M, Napoli A, Zaccagna F, Cartocci G, Saba L, Menichini G, et al. Liver Metastases From Colorectal Cancer Treated With Conventional and Antiangiogenetic Chemotherapy: Evaluation With Liver Computed Tomography Perfusion and Magnetic Resonance Diffusion-Weighted Imaging. J Comput Assist Tomogr (2011) 35(6):690-6. doi: 10.1097/RCT. 0b013e318230d905

8. Van Cutsem E, Verheul HM, Flamen P, Rougier P, Beets-Tan R, GlynneJones R, et al. Imaging in Colorectal Cancer: Progress and Challenges for the Clinicians. Cancers (Basel) (2016) 8(9):81. doi: 10.3390/cancers8090081

9. Hirashima Y, Yamada Y, Tateishi U, Kato K, Miyake M, Horita Y, et al. Pharmacokinetic Parameters From 3-Tesla DCEMRI as Surrogate Biomarkers of Antitumor Effects of Bevacizumab Plus FOLFIRI in Colorectal Cancer With Liver Metastasis. Int J Cancer (2012) 130(10):2359_ 65. doi: $10.1002 /$ ijc. 26282

10. Granata V, Fusco R, Catalano O, Filice S, Amato DM, Nasti G, et al. Early Assessment of Colorectal Cancer Patients With Liver Metastases Treated With Antiangiogenic Drugs: The Role of Intravoxelincoherent Motion in Diffusion-Weighted Imaging. PloS One (2015) 10(11):e0142876. doi: 10.1371/ journal.pone. 0142876

11. Hutchinson LG, Mueller HJ, Gaffney EA, Maini PK, Wagg J, Phipps A, et al. Modeling Longitudinal Preclinical Tumor Size Data to Identify Transient

\section{FUNDING}

We receive funds from LILT (lega Italiana per la Lotta contro i Tumori) for open access publication fees.

\section{ACKNOWLEDGMENTS}

We acknowledge the LILT (Lega Italiana per la Lotta contro i Tumori-sezione di Napoli) for the support and collaboration.

Dynamics in Tumor Response to Antiangiogenic Drugs. CPT Pharmacometrics Syst Pharmacol (2016) 5(11):636-45. doi: 10.1002/ psp4.12142

12. O'Connor JP, Carano RA, Clamp AR, Ross J, Ho CC, Jackson A, et al. Quantifying Antivascular Effects of Monoclonal Antibodies to Vascular Endothelial Growth Factor: Insights From Imaging. Clin Cancer Res (2009) 15(21):6674-82. doi: 10.1158/1078-0432.CCR-09-0731

13. Avallone A, Pecori B, Bianco F, Aloj L, Tatangelo F, Romano C, et al. Critical Role of Bevacizumab Scheduling in Combination With Pre-Surgical ChemoRadiotherapy in MRI-Defined High-Risk Locally Advanced Rectal Cancer: Results of the BRANCH Trial. Oncotarget (2015) 6(30):30394-407. doi: $10.18632 /$ oncotarget. 4724

14. Guinney J, Dienstmann R, Wang X, de Reynies A, Schlicker A, Soneson C, et al. The Consensus Molecular Subtypes of Colorectal Cancer. Nat Med (2015) 21(11):1350-6. doi: 10.1038/nm.3967

15. Alidzanovic L, Starlinger P, Schauer D, Maier T, Feldman A, Buchberger E, et al. The VEGF Rise in Blood of Bevacizumab Patients Is Not Based on Tumor Escape But a Host-Blockade of VEGF Clearance. Oncotarget (2016) 7 (35):57197-212. doi: 10.18632/oncotarget.11084

16. Greening DW, Lee ST, Ji H, Simpson RJ, Rigopoulos A, Murone C, et al. Molecular Profiling of Cetuximab and Bevacizumab Treatment of Colorectal Tumours Reveals Perturbations in Metabolic and Hypoxic Response Pathways. Oncotarget (2015) 6(35):38166-80. doi: 10.18632/oncotarget.6241

17. Wei TT, Lin YT, Tang SP, Luo CK, Tsai CT, Shun CT, et al. Metabolic Targeting of HIF-1 $\alpha$ Potentiates the Therapeutic Efficacy of Oxaliplatin in Colorectal Cancer. Oncogene (2020) 39(2):414-27. doi: 10.1038/s41388-0190999-8

Conflict of Interest: The authors declare that the research was conducted in the absence of any commercial or financial relationships that could be construed as a potential conflict of interest.

Publisher's Note: All claims expressed in this article are solely those of the authors and do not necessarily represent those of their affiliated organizations, or those of the publisher, the editors and the reviewers. Any product that may be evaluated in this article, or claim that may be made by its manufacturer, is not guaranteed or endorsed by the publisher.

Copyright (c) 2021 Ottaiano and Caraglia. This is an open-access article distributed under the terms of the Creative Commons Attribution License (CC BY). The use, distribution or reproduction in other forums is permitted, provided the original author (s) and the copyright owner(s) are credited and that the original publication in this journal is cited, in accordance with accepted academic practice. No use, distribution or reproduction is permitted which does not comply with these terms. 